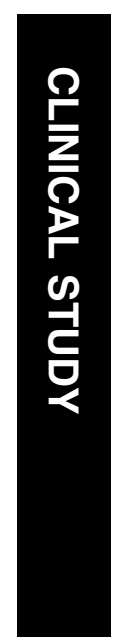

\section{Vitreo-papillary adhesion as a prognostic factor in pseudo- and lamellar macular holes}

MR Romano1,2, JL Vallejo-Garcia', FI Camesasca1, P Vinciguerra ${ }^{1}$ and C Costagliola ${ }^{2}$
${ }^{1}$ Department of Ophthalmology, Istituto Clinico Humanitas, Rozzano, Italy

${ }^{2}$ Department of Ophthalmology, University of Molise, Campobasso, Italy

Correspondence: MR Romano, Department of Ophthalmology, Istituto Clinico Humanitas, Via Manzoni, Rozzano, Milan 86100 Italy Tel: + 390255182463; Fax: +390823363005 . E-mail: romanomario@ email.it

Received: 15 July 2011 Accepted in revised form: 7 February 2012

Published online: 16 March 2012

This work was presented as a paper at the Club Gonin Meeting 2010 in Kyoto.

\begin{abstract}
Purpose To determine the incidence of vitreopapillary adhesion (VPA) and to investigate its value as a prognostic factor in the surgical outcome of pseudo- (PMH) and lamellar macular holes (LMH).

Methods A total of 76 consecutive patients, diagnosed with PMH (41 eyes) or LMH (35 eyes) were included. Eyes with VPA were alternatively assigned to the surgical or control group. Surgery consisted of a 25G vitrectomy and internal limiting membrane peeling with blue dye staining. There were six visits: baseline, the day of surgery, and 1, 3, 6, and 9 post-operative months. Main outcome measures were the incidence of VPA and changes in the outer retinal layers and visual acuity.

Results VPA was found in $27 \%(11 / 41)$ of patients with PMH and $37 \%$ (13/35) with LMH $(P=0.03)$. In presence of VPA, the best-corrected visual acuity (BCVA) improved in the surgery group from $32 \pm 8$ to $47 \pm 8$ letters, whereas the control group went from $34 \pm 7$ to $31 \pm 8$ letters. The difference in letters between the surgery and control groups was statistically significant for both distance $(P=0.032)$ and near $(P=0.04)$ vision. Intra-retinal cysts were significantly correlated with a poor functional prognosis $(P=0.01)$. We found the presence of focal damage to the outer retinal layers in LMH and PMH.

Conclusion VPA is more frequent in the presence of LMH vs PMH. It significantly influences the tangential forces at the vitreoretinal interface, exacerbating anatomical changes, and worsening the functional prognosis. Functional difference between the surgical and control groups was
\end{abstract}

statistically significant for distant and near vision in presence of VPA.

Eye (2012) 26, 810-815; doi:10.1038/eye.2012.43; published online 16 March 2012

Keywords: vitreo-papillary adhesion; pseudomacular hole; lamellar macular holes; epiretinal membrane

\section{Introduction}

Vitreopapillary adhesion (VPA) is defined as a prominent vitreous membrane attached to the borders of the optic disc. ${ }^{1}$ A possible pathogenic mechanism by which VPA contributes to tangential epimacular traction formation involves anomalous posterior vitreous detachment and vitreoschisis. ${ }^{2,3}$ Persistent adherence of vitreous to the optic disc may influence the vectors of force exerted on the macular interface. ${ }^{4}$ We hypothesize that in the presence of vitreoschisis, adhesion of vitreous to the optic disc ultimately evolves into traction, with possible damage to the outer retinal layers even in the presence of pseudo- (PMH) and lamellar macular holes (LMH).

The aim of this study was to evaluate the prevalence of VPA in PMH and LMH and to investigate the value of VPA as a prognostic factor in surgical outcome. We also investigated changes in the outer and inner retinal layers.

Patients and methods

Study design

We conducted a prospective, randomized, comparative interventional study on 76 eyes of 75 consecutive patients diagnosed with a $\mathrm{PMH}$ (41 eyes) or LMH (35 eyes). 
The study complied with the policies of the University's Ethical Committee.

\section{Subjects}

To be eligible for enrollment and randomized treatment assignment, patients had to have a functional impairment (ie, a visual acuity (VA) decrease and/or metamorphopsia) as well as a diagnosis of $\mathrm{PMH}$ or $\mathrm{LMH}$ confirmed by spectral domain optical coherence tomography (SD-OCT) according to Witkin's classification. ${ }^{5}$

We excluded patients who were less than 18 years of age, who had severe systemic disease, any untreated ocular disease, myopia greater than or equal to 6 diopters, or any pregnant women.

All patients were recruited from $\mathrm{S}$. Sebastiano Hospital, Caserta, Italy; Clinica M. Rosaria, Pompei, Italy; and Istituto Clinico Humanitas, Rozzano, Italy between September 2008 and December 2009. The local Human Subjects Review Committee approved the study.

\section{Examinations}

Pre-and post-operative examinations included a detailed ophthalmic history, refracted best-corrected visual acuity (BCVA; Sloan ETDRS near and distance vision charts), slit lamp biomicroscopy, Goldman tonometry, and SD-OCT. The study protocol included a minimum of six clinical visits: baseline, the day of surgery, and 1, 3, 6, and 9 post-operative months.

\section{Optical coherence tomography}

OCT scans were obtained using a Cirrus SD highdefinition OCT (Carl Zeiss Meditec AG, Jena, Germany) and RTvue-100 OCT system (Optovue Inc., Fremont, CA, USA). In each patient, raster line scans of the macular area/optic nerve and macular cube scans were performed.

Linear SD-OCT scanning was also used to identify VPA, defined as a prominent vitreous membrane attached to the borders of the optic disc.

OCT patterns evaluated for statistical analysis were the presence of intra-retinal cysts and the four post-operative patterns regarding the integrity of the external limiting membrane (ELM) and inner and outer (IS/OS) junction: (1) ELM-IS/OS integrity; (2) ELM integrity-IS/OS interruption; (3) ELM interruption-IS/OS integrity and (4) ELM-IS/OS interruption.

\section{Randomization}

Patients with VPA who fulfilled the inclusion and exclusion criteria signed the informed consent form, were assigned an identification number that was recorded on the baseline data forms, and were then randomly assigned to the surgical or control group. Randomization was performed by random block permutation according to a computer-generated randomization list.

\section{Interventions}

Pseudophakia was performed before pars plana vitrectomy under general anesthesia or with a retrobulbar block. The non-contact wide-angle fundus observation system EIBOS (Moeller-Wedel OPTICAL GmbH, Wedel, Germany) was used in all cases. The surgical procedure included a 25-gauge three-port pars plana vitrectomy and ILM peeling with blue-dye assistance.

The epiretinal membrane and ILM were removed with either Membrane Blue or Brilliant Peel (DORC International, Rotterdam, the Netherlands). In both groups, the vitreous base was thoroughly trimmed during vitrectomy. The retinal periphery was inspected for retinal breaks that were treated with endolaser photocoagulation. A fluid-air exchange was then performed with humidified air. No prone positioning was advised after surgery.

\section{Outcome measures}

The primary endpoint was visual function (near and distance) and the ultrastructural changes of the outer retinal layers. Secondary endpoints were the prevalence of VPA and intra-retinal cysts.

\section{Statistical methods}

Statistical analysis was performed with SPSS software, version 13.0 (SPSS, Inc., Chicago, IL, USA). The Student's $t$-test was used to compare the mean change in VA from baseline to 9 months for each patient, as well as a parallel analysis between the two groups at each time point. The level of significance was set at 0.05 . The statistical significance of differences for categorical data between two groups was evaluated with a one-way ANOVA test. The significance of correlations between variables was assessed using the Pearson correlation coefficient and Spearman's rank correlation coefficient.

\section{Results}

Patients and pre-operative findings

In all, 41 eyes with PMH (age: $64 \pm 11$ years; 22 males/ 19 females) and 35 eyes with LMH (age: $61 \pm 14$ years; 19 males/16 females) were included in the study. A total 
of 24 eyes were found to have a VPA (11 PMH and $13 \mathrm{LMH}$ ) and were then randomly assigned to either the surgical (15 eyes) or control group (9 eyes).

The prevalence of VPA was $27 \%(11 / 41)$ in eyes with $\mathrm{PMH}$ and $37 \%(13 / 35)$ in eyes with $\mathrm{LMH}(P=0.04)$.

In the presence of VPA, the prevalence of intra-retinal cysts was $64 \%(7 / 11)$ in eyes with $\mathrm{PMH}$ and $46 \%(6 / 13)$ in eyes with LMH. In contrast, in the absence of VPA, the overall prevalence of intra-retinal cysts was 9.6\% (5/52). Intra-retinal cysts were significantly correlated with a poor functional prognosis $(P=0.01)$ at the 9 -month follow-up visit.

\section{Functional results}

Figure 1 illustrates the VA changes throughout the 9-month follow-up period. Regarding distance vision from baseline to 9 months, the BCVA improved in the surgery group from $32 \pm 8$ to $47 \pm 8$ letters $(P=0.037)$, whereas the control group went from $34 \pm 7$ to $31 \pm 8$ letters $(P=0.2)$.

Regarding near vision from baseline to 9 months, the BCVA in the surgery group improved from $24 \pm 11$ to $35 \pm 10$ letters $(P=0.054)$, whereas the control group was essentially unchanged, from $21 \pm 9$ to $20 \pm 12$ letters $(P=0.6)$. The difference in letters between the surgery and control groups was statistically significant for both distance $(P=0.032)$ and near $(P=0.04)$ vision.

\section{Outer retinal layers}

Figure 2 illustrates the four patterns of outer retinal layer changes throughout the 9-month follow-up period. In the surgery group, the percentage of ELM-IS/OS integrity improved from 67 to $80 \%$; the ELM integrity-IS/OS interruption was reduced from 20 to $14 \%$; the ELM

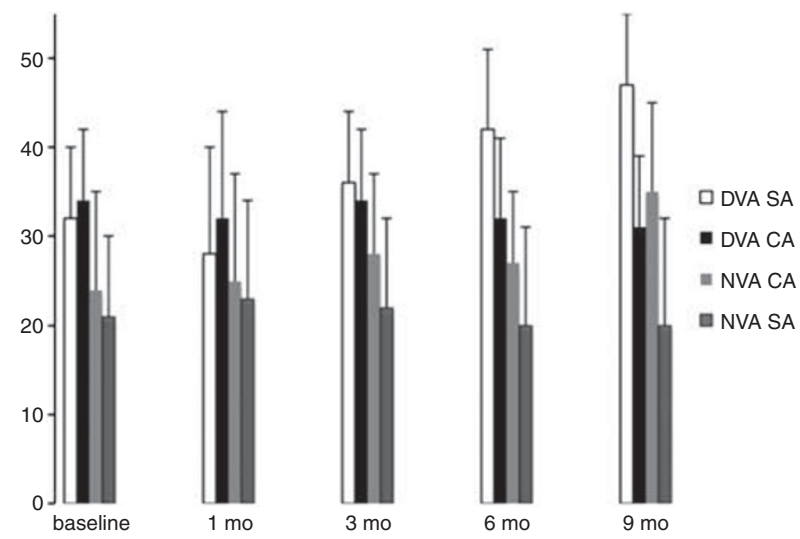

Figure 1 Mean changes in VA (in letters) throughout the 9-month follow-up period. At last follow-up, the difference between the surgical arm (SA) and control arm (CA) was statistically significant for distant VA (DVA, $P=0.032$ ) and near VA (NVA, $P=0.04)$.
interruption-IS/OS integrity went from 7 to $0 \%$, and the ELM-IS/OS interruption was unchanged. In the control group, the percentage of ELM-IS/OS integrity was unchanged; the ELM integrity-IS/OS interruption went from 22 to $11 \%$; the ELM interruption-IS/OS integrity went from 0 to $11 \%$, and the ELM-IS/OS interruption was unchanged.

The differences of ELM-IS/OS integrity patterns between the surgery and control groups at last follow-up were not statistically significant (one-way ANOVA).

The integrity of the ELM and outer segment (IS/OS) was not significantly correlated with BCVA $(P=0.02$, Spearman correlation) at 9 months.

\section{Discussion}

VPA, defined as persistent adherence of an anomalous posterior vitreous detachment to the optic disc, is known to induce epiretinal traction and intraretinal changes such as cysts (Figure 3). ${ }^{1,4}$ Advanced synchysis of vitreous gel can also induce vitreoschisis. ${ }^{6}$ The outer layer of vitreous cortex left on the retinal surface can lead to tangential traction and the formation of a macular hole and/or macular pucker. ${ }^{2}$ We believe that in LMH and $\mathrm{PMH}$, the presence of vitreoschisis and a strong adhesion of vitreous cortex to the optic disc enhances the force of the tangential vector and thus leads to progression of an $\mathrm{LMH}$ and PMH up to the outer retinal layers. This is a similar mechanism to the one described for macular pucker and macular hole. ${ }^{1,4}$

Haouchine $\mathrm{et}^{7} \mathrm{l}^{7}$ proposed a differential diagnosis of $\mathrm{LMH}$ and $\mathrm{PMH}$ based on OCT findings, such as steepening of the foveal pit, central foveal thickness, splitting or thickness of foveal edges, and a small foveal

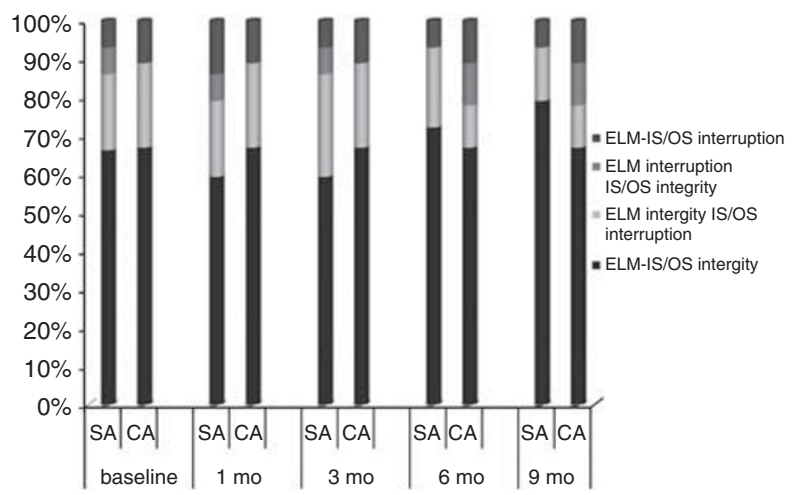

Figure 2 The stacked histogram bar is divided in percent according to the four patterns of outer retinal changes throughout the 9-month follow-up period. A slight and not significant decrease in the IS/OS interruption patterns was recorded in the surgery group (SA). ELM-IS/OS integrity patterns were not statistically significantly different between the surgical and control group (CA) at last follow-up. 

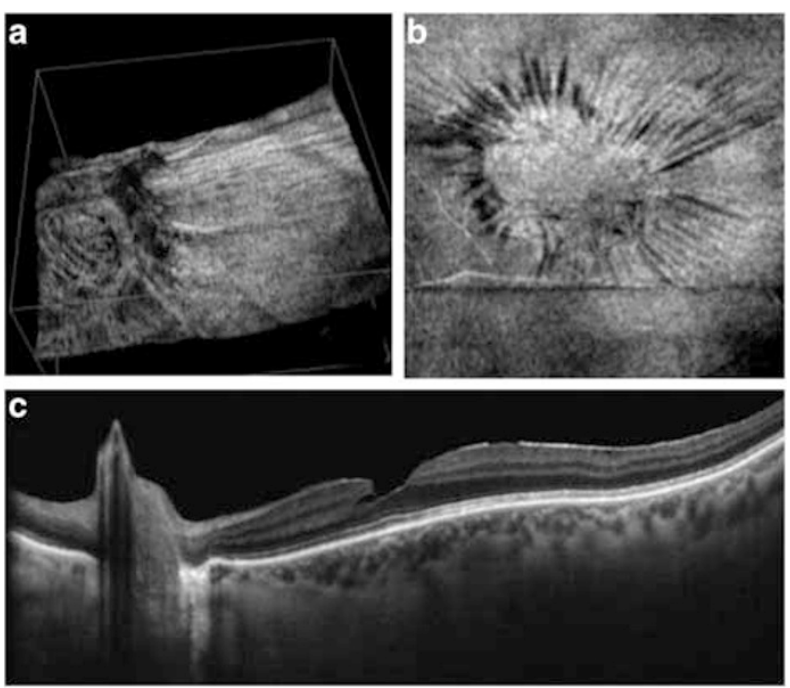

Figure 3 The vitreopapillary adhesion, defined as a prominent vitreous membrane attached to the borders of the optic disc (a), enhances the tangential traction on the macula (b). The intraretinal split (c), observed between the outer plexiform layer and the outer nuclear layer, is in line with the traction induced by the vitreopapillary adhesion.

pit diameter. Witkin et al ${ }^{8}$ introduced new parameters in the diagnosis of LMH based on an irregular foveal contour, a break in the inner fovea, dehiscence of the inner foveal retina from the outer retina, and an absence of a full thickness foveal defect with intact foveal photoreceptors. Hence, LMH and PMH are currently described as defects of the inner retina with intraretinal cystoid spaces, sometimes associated with epiretinal membrane formation, in the presence of intact outer retinal layers and an undamaged photoreceptor layer. Our findings differ with previous descriptions of PMH and $\mathrm{LMH}^{7,8}$ as we have demonstrated the presence of focal damage to the outer retinal layers: the ELM and the IS/OS junction (Figure 4).

The ELM, formed by continuous bands of intermediate junctions that link the photoreceptor nuclei to Müller cells, and the connecting cilium (IS/OS junction) between the inner and outer segment of the photoreceptor, are well illustrated by SD-OCT. ${ }^{9}$ The integrity of the ELM, rather than the integrity of the IS/OS junction, might better reflect the integrity and function of the whole photoreceptor. ${ }^{10}$ If restoration of the photoreceptor layer can occur in cases in which degenerative changes have not yet reached the photoreceptor cell bodies, the damage to the IS/OS junction may be reversible, whereas integrity of the ELM seems critical for predicting the potential restoration of the photoreceptor microstructures. ${ }^{11}$ This is particularly important in LMH, where the intraretinal separation occurs between the outer plexiform layer and the outer nuclear layer, next to
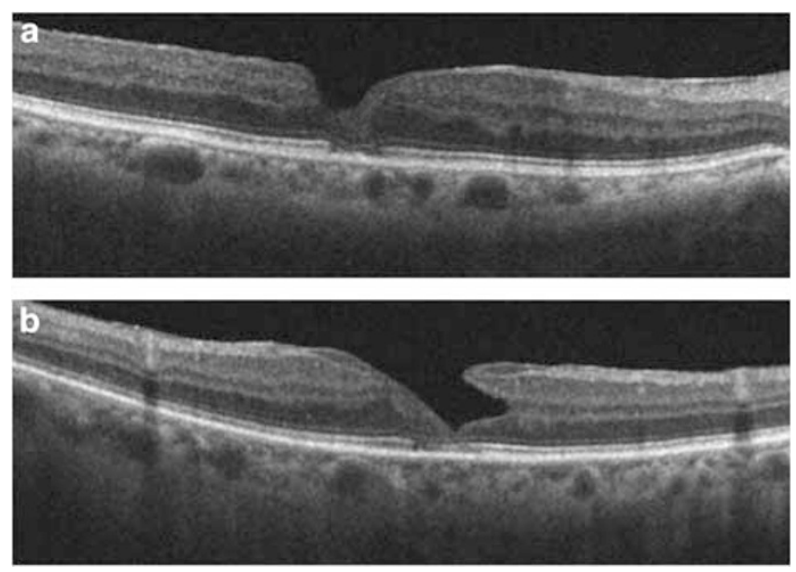

Figure 4 Damage of outer retinal layers. A pseudo-macular hole (a) and lamellar macular hole (b) can be observed with integrity of the ELM and interruption of the IS/OS junction.

the photoreceptor cell bodies. In the presence of further traction, such as that caused by VPA, visual function can be damaged more rapidly and irreversibly. In our series, in the presence of VPA, we reported integrity of both the ELM and the IS/OS junction only in $67 \%$ of cases.

Our findings are supported by a recent longitudinal study of LMH with a 3-year follow-up, where the diameter of LMH was found to increase by an average of $13.7 \%$ and the BCVA deteriorated in $22 \%$ of the cases. ${ }^{12}$ The presence of tangential epiretinal traction was proven to deteriorate the VA and to be a negative prognostic factor. ${ }^{4}$ In this dynamic process, the size of the foveal inner layer defect seems to have less influence on the functional outcome than defects in the photoreceptor layer. ${ }^{13}$

Surgical removal of the ERM-ILM in PMH and LMH remains controversial, with respect to functional results and the foveal appearance on OCT scans. ${ }^{5,13}$ The microstructural damage to the IS/OS junction, caused by traction and visible by $\mathrm{OCT}$, indicates that outer retinal layer changes cannot be attributed entirely to apoptosis, and that probably the recovery visible by OCT after ILM peeling is due to a reduction in photoreceptor displacement induced by the swelling of glial cells in the presence of traction.

Garretson $e a^{14}$ investigated retrospectively $27 \mathrm{LMH}$ treated with ILM peeling and described an overall functional improvement of 3.2 Snellen lines after surgery. Engler $e t a l^{15}$ reported a retrospective study on 10 eyes with LMH treated with ERM-ILM peeling and tamponade with gas (only one with oil). With a minimum follow-up of 6 months, the BCVA gain was $3 \pm 2$ lines (from 0.35 to $0.64 \log M A R$ ). Michalewska et $a l^{13}$ recently reported a prospective, non-comparative study on 26 patients diagnosed with LMH and treated 
with ERM-ILM peeling without the use of endotamponade. At 12 months after surgery, the mean BCVA improved from 0.2 to 0.51 , with favorable functional results in 24 out of 26 eyes. The authors also showed encouraging anatomical results, with disappearance of the IS/OS defects in 6 out of 8 cases. Conversely, Witkin et $a l^{5}$ did not find any significant functional result after membrane peeling in a retrospective series of 16 eyes with $\mathrm{LMH}$. The authors reported that postoperatively 2 eyes (13\%) developed full-thickness macular holes and 6 eyes (38\%) continued to have an $\mathrm{LMH}$.

As opposed to previously published studies, we randomized only patients with LMH associated to VPA, as this is a sign of stronger epiretinal tangential traction, confirmed by the presence of intraretinal cysts. Following this surgical inclusion criterion, we recorded a mean improvement in the surgical group of three lines for distance vision (from $32 \pm 8$ to $47 \pm 8 ; P=0.037$ ) and of two lines for near vision (from $24 \pm 11$ to $35 \pm 10$; $P=0.054)$ at the 9-month follow-up. The functional difference between the surgical and control groups was statistically significant for distant and near vision ( $P=0.032$ and $P=0.04$, respectively).

There are some limitations to our study, such as the number of patients and the limited follow-up period of 9 months. Further prospective randomized trials are warranted to confirm our findings.

From the analysis of the anatomical results, as seen on SD-OCT scans, as well as the functional outcomes of our series, we propose a new classification of PMH and LMH formation, according to three different patterns (Figure 5):

- Type 1. The omega-shaped LMH is the final step of foveal pseudocyst interruption. ${ }^{16-19}$ This pattern is not progressive and comes from a full-thickness posterior vitreous detachment with a prevalent anteriorposterior traction.

- Type 2. PMH or LMH with epiretinal membrane. The presence of an epiretinal membrane induces progressive changes, with or without VPA, transforming one form into the other. ${ }^{20}$ The diagnosis of $\mathrm{PMH}$ or $\mathrm{LMH}$ is probably only time dependent. SD-OCT scans show $\mathrm{PMH}$ with intra-retinal cysts and active tangential traction that induces an intraretinal split towards an $\mathrm{LMH}$. This pattern is progressive and comes from a vitreoschisis anterior to the level of hyalocytes, inducing a tangential multifocal contraction, and thus damaging the outer retinal layers. The presence of VPA increases the risk of progression.

- Type 3. LMH without epiretinal membrane. The abortive process of macular hole formation due to a centrifugal contraction ${ }^{4}$ is caused by the presence
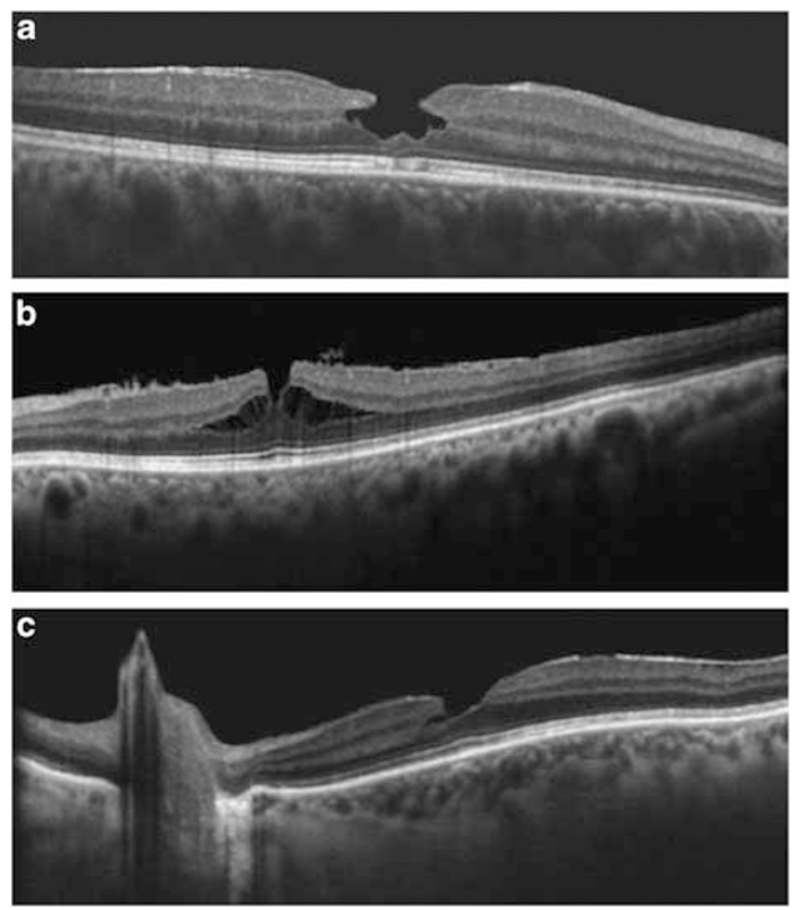

Figure 5 Lamellar macular hole formation. (a) Type 1. The omega-shape LMH is a final step in foveal pseudocyst interruption. This pattern is not progressive and comes from a full-thickness posterior vitreous detachment (PVD) with significant antero-posterior traction. (b) Type 2. The presence of an epiretinal membrane induces progressive changes, with or without vitreopapillary adhesion, transforming one form into the other. The diagnosis of PMH or LMH is probably only time dependent. The SD-OCT scan illustrates an PMH with an intraretinal cyst and an active tangential traction that induced an intra-retinal split towards the LMH. This pattern is progressive and comes from a vitreoschisis anterior to the hyalocytes, thus inducing a tangential multifocal contraction that damages the outer retinal layers. (c) Type 3. The abortive process of macular hole formation due to a centrifugal contraction, in the presence of vitreoschisis posterior to hyalocytes, without an epiretinal membrane. This pattern is not progressive, except in the presence of a vitreopapillary adhesion (c). Its progression can induce outer retinal layer damage.

of vitreoschisis posterior to the level of hyalocytes. This pattern is not progressive, except in the presence of VPA. This progression can induce outer retinal layer damage.

In conclusion, our findings show that even partial retinal defects, such as $\mathrm{PMH}$ and $\mathrm{LMH}$, can cause damage to the outer retinal layers and permanently compromise visual function. The presence of VPA significantly influences the tangential forces at the vitreoretinal interface, inducing a progression of anatomical changes up to the outer retinal layers, visible by SD-OCT scans, and worse functional results regarding 
both distant and near vision. VPA is more frequent in the presence of $\mathrm{LMH}$ than in presence of $\mathrm{PMH}$. In the presence of VPA, surgical removal of the epiretinal traction does lead to better visual results.

\section{Summary}

What was known before

- The outer layer of vitreous cortex left on the retinal surface can induce a tangential traction involved in the formation of macular hole and macular pucker.

\section{What this study adds}

- In the presence of vitreoschisis, adhesion of vitreous at the optic disc (vitreopapillary adhesion) becomes traction, with possible damage to the outer retinal layers even in pseudo- and lamellar macular holes.

\section{Conflict of interest}

The authors declare no conflict of interest.

\section{References}

1 Sebag J, Wang MY, Nguyen D, Sadun AA. Vitreopapillary adhesion in macular diseases. Trans Am Ophthalmol Soc 2009; 107: $35-44$

2 Sebag J, Gupta P, Rosen RR, Garcia P, Sadun AA. Macular holes and macular pucker: the role of vitreoschisis as imaged by optical coherence tomography/scanning laser ophthalmoscopy. Trans Am Ophthalmol Soc 2007; 105: 121-129; discusion 129-131.

3 Johnson MW. Posterior vitreous detachment: evolution and complications of its early stages. Am J Ophthalmol 2010; 149: 371-382.e1.

4 Wang MY, Nguyen D, Hindoyan N, Sadun AA, Sebag J. Vitreo-papillary adhesion in macular hole and macular pucker. Retina 2009; 29: 644-650.

5 Witkin AJ, Castro LC, Reichel E, Rogers AH, Baumal CR, Duker JS. Anatomic and visual outcomes of vitrectomy for lamellar macular holes. Ophthalmic Surg Lasers Imaging 2010; 41(4): 418-424.

6 Sebag J. Vitreoschisis. Graefes Arch Clin Exp Ophthalmol 2008; 246: 329-332.

7 Haouchine B, Massin P, Tadayoni R, Erginay A, Gaudric A. Diagnosis of macular pseudoholes and lamellar macular holes by optical coherence tomography. Am J Ophthalmol 2004; 138: 732-739.
8 Witkin AJ, Ko TH, Fujimoto JG, Schuman JS, Baumal CR, Rogers $\mathrm{AH}$ et al. Redefining lamellar holes and the vitreomacular interface: an ultrahigh-resolution optical coherence tomography study. Ophthalmology 2006; 113: 388-397.

9 Wojtkowski M, Srinivasan V, Fujimoto JG, Ko T, Schuman JS, Kowalczyk A et al. Three-dimensional retinal imaging with high-speed ultrahigh-resolution optical coherence tomography. Ophthalmology 2005; 112: 1734-1746.

10 Ooto S, Hangai M, Takayama K, Sakamoto A, Tsujikawa A, Oshima S et al. High-resolution imaging of the photoreceptor layer in epiretinal membrane using adaptive optics scanning laser ophthalmoscopy. Ophthalmology 2010; 118(5): 873-881.

11 Inoue M, Morita S, Watanabe Y, Kaneko T, Yamane S, Kobayashi $S$ et al. Inner segment/outer segment junction assessed by spectral-domain optical coherence tomography in patients with idiopathic epiretinal membrane. Am J Ophthalmol 2010; 150: 834-839.

12 Theodossiadis PG, Grigoropoulos VG, Emfietzoglou I, Nikolaidis P, Vergados I, Apostolopoulos $\mathrm{M}$ et al. Evolution of lamellar macular hole studied by optical coherence tomography. Graefes Arch Clin Exp Ophthalmol 2009; 247: 13-20.

13 Michalewska Z, Michalewski J, Odrobina D, Pikulski Z, Cisiecki S, Dziegielewski K et al. Surgical treatment of lamellar macular holes. Graefes Arch Clin Exp Ophthalmol 2010; 248: 1395-1400.

14 Garretson BR, Pollack JS, Ruby AJ, Drenser KA, Williams GA, Sarrafizadeh R. Vitrectomy for a symptomatic lamellar macular hole. Ophthalmology 2008; 115: 884-886 e881.

15 Engler C, Schaal KB, Hoh AE, Dithmar S. Surgical treatment of lamellar macular hole. Ophthalmologe 2008; 105: 836-839.

16 Gass JD. Lamellar macular hole: a complication of cystoid macular edema after cataract extraction. Arch Ophthalmol 1976; 94: 793-800.

17 Gaudric A, Haouchine B, Massin P, Paques M, Blain P, Erginay A. Macular hole formation: new data provided by optical coherence tomography. Arch Ophthalmol 1999; 117: $744-751$

18 Takahashi H, Kishi S. Tomographic features of a lamellar macular hole formation and a lamellar hole that progressed to a full-thickness macular hole. Am J Ophthalmol 2000; 130: $677-679$

19 Haouchine B, Massin P, Gaudric A. Foveal pseudocyst as the first step in macular hole formation: a prospective study by optical coherence tomography. Ophthalmology 2001; 108: 15-22.

20 Michalewski J, Michalewska Z, Dziegielewski K, Nawrocki J. Evolution from macular pseudohole to lamellar macular hole - spectral domain OCT study. Graefes Arch Clin Exp Ophthalmol 2011; 249: 175-178. 